\title{
Alumnado con dificultades específicas de aprendizaje, políticas administrativas.
}

\section{Students with specific learning difficulties, administrative policies.}

\author{
Abraham Bernárdez Gómez \\ Departamento de pedagogía y didáctica, Universidad de Santiago de Compostela, España
}

\begin{abstract}
Resumen
El objetivo de esta investigación es analizar y reflexionar sobre las medidas de atención a la diversidad tomadas con un grupo específico de alumnado con necesidades específicas de apoyo educativo (ANEAE). Para alcanzar este objetivo se empleará una metodología Ex Post-facto de tipo descriptivo donde realizaremos un análisis de contenido de la documentación extraída de la Consellería de educación de la comunidad autónoma de Galicia. Este proceso de indagación en la documentación se llevará a cabo utilizando el programa de análisis cualitativo Atlas.Ti. De esta forma se ahondará en los puntos de referencia para la intervención en el alumnado.

Palabras clave: dificultades de aprendizaje, necesidades educativas especiales, programas especiales, intervención.
\end{abstract}

\begin{abstract}
The objective of this research is to analyze and reflect on measures of attention to diversity taken with a specific group of students with specific educational support needs (ANEAE). To reach this objective we will use a methodology Ex Post-facto descriptive type where we will perform a content analysis of the documentation extracted from the Consellería de educación of the Autonomous Community of Galicia. This process of inquiry in the documentation will be carried out using the Atlas.Ti qualitative analysis program. In this way we will delve into the benchmarks for intervention in students.

Keywords: Learning Disabilities, special education needs, special programs, intervention.
\end{abstract}

La diversidad en los centros educativos es un hecho, y por ello la atención a la diversidad tomada por el sistema educativo debe llevarse a cabo teniendo en cuenta los ritmos madurativos, diferencias intrínsecas o no, las diferentes variables por la que se ven afectados, etc., de cada uno de los alumnos.

La atención a la diversidad se lleva a cabo en los centros donde se presentan casos de alumnos con necesidades específicas de apoyo educativo, como las dificultades específicas de aprendizaje, trastornos por déficit de atención, condiciones personales especiales, incorporación tardía al sistema escolar o altas capacidades.

Con idea de indagar sobre este aspecto, nuestro objetivo de investigación será analizar y reflexionar sobre las medidas de atención a la diversidad tomadas con un grupo específico de alumnado con necesidades específicas de apoyo educativo, los que presentan dificultades específicas de aprendizaje.

\section{Método}

Cualquier investigación trata de aplicar el método científico para permitir al actuante obtener información relevante de un hecho y, de esta forma, ahondar en el conocimiento de un ámbito (Bisquerra, 2016). Así pues, teniendo por meta la indagación sobre la forma de intervenir recomendada desde la administración y así aproximarnos a la realidad de la atención a la diversidad en dicho ámbito. Realizamos el proceso de investigación utilizando los instrumentos descritos y el proceso que se expone a continuación.

\section{Instrumentos}

Para llevar a cabo la investigación de la que aquí se da cuenta se ha empleado la documentación disponible en: http://www.edu.xunta.gal/portal/diversidadeorientacion/ $141 / 145$. Se trata de un manual de 50 hojas perteneciente a la colección "Orientación e Respostas educativas", que trata de ilustrar a profesorado y familias sobre los aspectos básicos a tener en cuenta sobre las dificultades específicas de aprendizaje.

\section{Procedimiento}

Una vez localizada la documentación con la que se trabajó, accedimos a ella en la página web mencionada y se procedió a realizar una "descripción precisa y cuidadosa de este fenómeno educativo" (Bisquerra, 2016) para poder dar respuesta al objetivo planteado al inicio de esta investigación. Una vez analizada se expusieron los puntos más relevantes y se elaboró la discusión que figura.

\section{Resultados}

En esta investigación, lo que nos interesa es conocer como señala la administración que se debe atender a un colectivo de alumnos que figuran dentro del alumnado denominado de necesidades específicas de apoyo educativo (NEAE), el alumnado con dificultades específicas de aprendizaje. Como señalamos anteriormente, en estas líneas encontramos lo que expone la colección de Orientación y Respuestas Educativas sobre este alumnado. Para seguir describir fielmente dichas recomendaciones de actuación, se expone 
teniendo en consideración las pautas de la fuente empleada. Por ello empleamos cuatro puntos sobre los que pivota la acción de atención a la diversidad: el conocimiento del concepto, los identificadores y aspectos para valorar la intervención del profesorado de apoyo, la respuesta educativa adecuada desde el centro y la relación necesaria con las familias.

El concepto. El texto que nos encontramos aborda esta NEAE desde el marco del Manual DSM-IV (APA, 2000) y el CIE-10 (OMS, 1992), por lo que es la principal fuente de conocimiento de la que se nutre. Así pues, empieza ofreciéndonos una definición del concepto para comenzar a situar al lector sobre la problemática de este alumnado.

Rendimiento escolar por debajo de lo esperado en las capacidades para la lectura, el cálculo y/o la expresión escrita -teniendo en cuenta la edad cronológica del alumnado, su escolarización y su nivel intelectual- que interfiere negativamente en el desarrollo de su proceso curricular y/o en las actividades cotidianas que requieren adquisición de dichas destrezas. (Xunta de Galicia, 2008. p.7)

De ella cabe señalar los distintos puntos que se abordan y su heterogeneidad, desde las distintas variables que afectan a las capacidades de bajo rendimiento hasta el mismo proceso de desarrollo del alumnado. En un primer lugar diferenciamos esos cuatro trastornos que integran las dificultades específicas de aprendizaje (DEA). (Véase Figura 1)

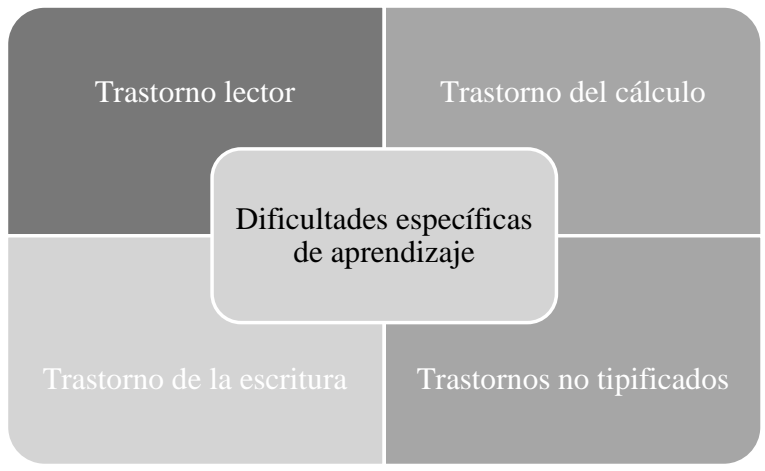

Figura 1: Trastornos que integran las DEA.

De los cuatro señalados, tenemos que los referentes a la lectura, escritura y cálculo están concretados y diferenciados de otras dificultades que se muestran en el periodo de escolaridad. Como podemos observar a través de su definición, este trastorno aparece relacionado con otros factores a tener en cuenta, como el nivel intelectual o edad cronológica del alumnado. Sin embargo, se insiste en señalar esa diferencia entre otras dificultades con las que se señala comorbilidad, como el atraso mental, alteraciones visuales auditivas o trastorno de la comunicación. Para concretar la idea de las áreas afectadas, podemos encontrar desgranado cada uno de los trastornos involucrados (Véase Tabla 1).
Tabla 1.

Características de los trastornos.

\begin{tabular}{lll}
\hline \multicolumn{1}{c}{ Lectura } & \multicolumn{1}{c}{ Cálculo } & \multicolumn{1}{c}{ Escritura } \\
\hline - Exactitud & $\bullet$ Procesos básicos & $\bullet$ Disgrafía \\
- Velocidad & $\bullet$ Sistema numérico & $\bullet$ Disortografía \\
- Comprensión & • Cálculos numéricos & $\bullet$ Expresión y \\
& - Lectura simbólica & composición \\
& - Resolución de & \\
& problemas & \\
\hline
\end{tabular}

Además, para cada uno de ellos se indican unos criterios a emplear para establecer un diagnostico en cada uno de estos trastornos:

- Trastorno lector. Se valorará el rendimiento mediante pruebas de precisión y/o comprensión individuales con el fin de situar cronológicamente su nivel de capacidad. Con ello se deberá valorar si interfiere en el rendimiento académico y si existe déficit sensorial que se vea aumentado por esta dificultad.

- Trastorno del cálculo. Teniendo en cuenta la capacidad evaluada mediante pruebas normalizadas. Se estimará la incidencia de ésta en la vida escolar y cotidiana del alumno. Además de tener en cuenta si existen otros déficits y su nivel de afectación.

- Trastorno de la escritura. Al igual que las anteriores, se realizará una evaluación mediante pruebas normalizadas de las habilidades funcionales para la escritura y se tendrá en cuenta la comorbilidad con otras alteraciones.

- Trastorno del aprendizaje no especificado. Donde se valoran los déficits que no alcanzan niveles significativos para enmarcarlos dentro de los ya señalados o los que hacen referencia a un déficit en el rendimiento escolar de manera generalizada.

Una vez explicados los aspectos fundamentales de los trastornos del aprendizaje, el texto se centra en las distintas hipótesis que le intentan dar explicación. Por un lado tenemos el punto de vista de la teoría cognitivista que desarrollan una explicación mediante un proceso (Véase Figura 2) en el que los estímulos del exterior acceden al proceso de aprendizaje y se incorporan en el tratamiento de la memoria. 


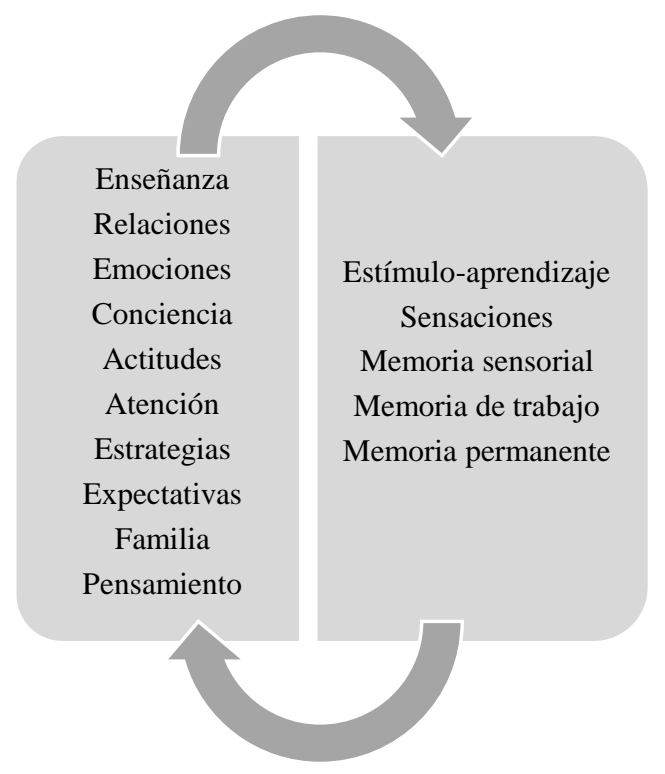

Figura 2: Proceso de la teoría cognitivista.

Por otro lado, también se muestra la importancia de los modelos del aprendizaje mediado (Véase Figura 3), que complementan a los anteriores. Estos consideran a la persona un todo donde las distintas variables intervienen en el proceso de aprendizaje.

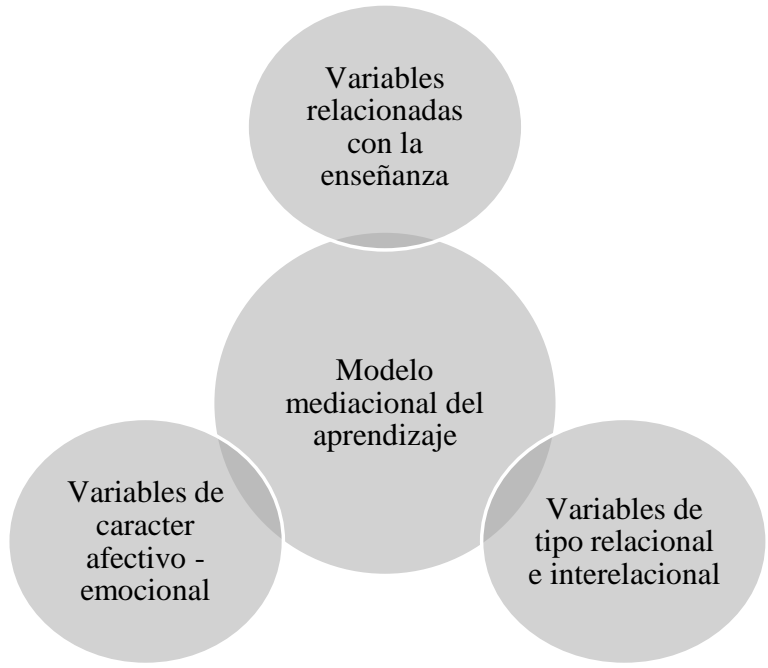

Figura 3: Variables en el modelo mediacional de aprendizaje.

Identificadores y valoración. En una segunda parte del texto que empleamos para hacer el análisis se nos invita a conocer el proceso recomendado para la identificación y valoración de los trastornos mencionados. Como podemos comprobar a través de la exposición que se realiza en el escrito, la principal vía de actuación es la identificación de las necesidades en los distintos ámbitos a intervenir, focalizando la acción en el individuo (Véase Figura 4). De esta forma contempla la evaluación a nivel institucional de centro y aula, con la posterior valoración de los distintos aspectos a tener en cuenta para el alumno, desde los comunes a todos los trastorno hasta los específicos de cada uno de ellos.

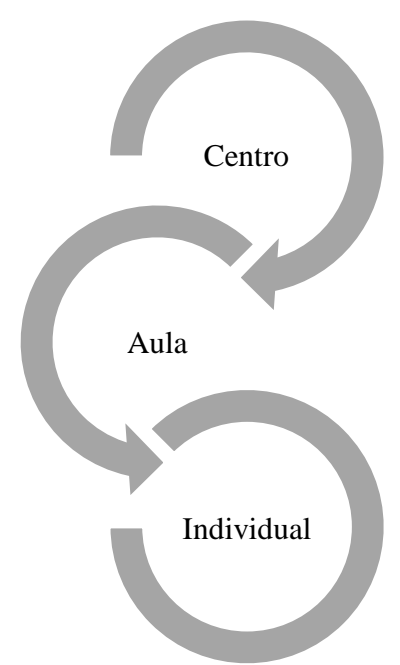

Figura 4: Niveles de valoración.

A modo de síntesis, nos indican que esta evaluación debe aportar información sobre una serie de aspecto clave: los conocimientos previos del alumno, los errores específicos u observables, las habilidades para relacionar contenidos aprendidos con los nuevos, las distintas estrategias cognitivas y metacognitivas, y las estrategias de regulación y supervisión del aprendizaje.

Respuesta educativa. En el punto donde ahora nos situamos, aparecen una serie de pistas que nos pondrán en camino de la intervención desde el punto de vista de la comunidad educativa, en concreto, por parte del profesorado. Se comienza señalando la importancia de priorizar los objetivos relacionados con la creación de estrategias de comprensión para establecer una vía eficiente de construcción de conocimiento. De esta forma se dará prioridad al establecimiento de procesos metodológicos y organizativos para favorecer la atención a la diversidad de los alumnos.

Al igual que en el punto anterior, se realiza una síntesis de los aspectos concretos, para los alumnos, en las que se debería definir el proceso de aprendizaje:

- Disponer de los conocimientos previos que les permitan establecer conexiones con las nuevas competencias a adquirir. Para ello se debe tener en cuenta en todo momento el punto de partida de los alumnos.

- Estimular el proceso metacognitivo para hacer consciente al alumno de las propias capacidades y establecer un plan para la consecución de estas. Estas estrategias y procesos deberán ser evaluadas bidireccionalmente de forma sistematizada con el fin de realizar las modificaciones pertinentes.

Para todo ello se elaborará un enfoque holístico de actuación con tres niveles de competencias diferenciados: uno de aplicación y aprendizajes, con actividades autónomas; otro de logro de objetivos específicos desarrollando las capacidades aprendidas; y un tercero de capacidad de respuesta a situaciones y problemas complejos fomentando la utilización de competencias generales.

Por otro lado, desde el punto de vista didáctico, al docente se le recomienda caracterizar su acción por cuatro modelos básicos (Véase Figura 5). A través de 
ellos podemos inferir esa inclinación para actuar sobre todos los frentes, poniendo el acento en una metodología centrada en la acción de los estudiantes.

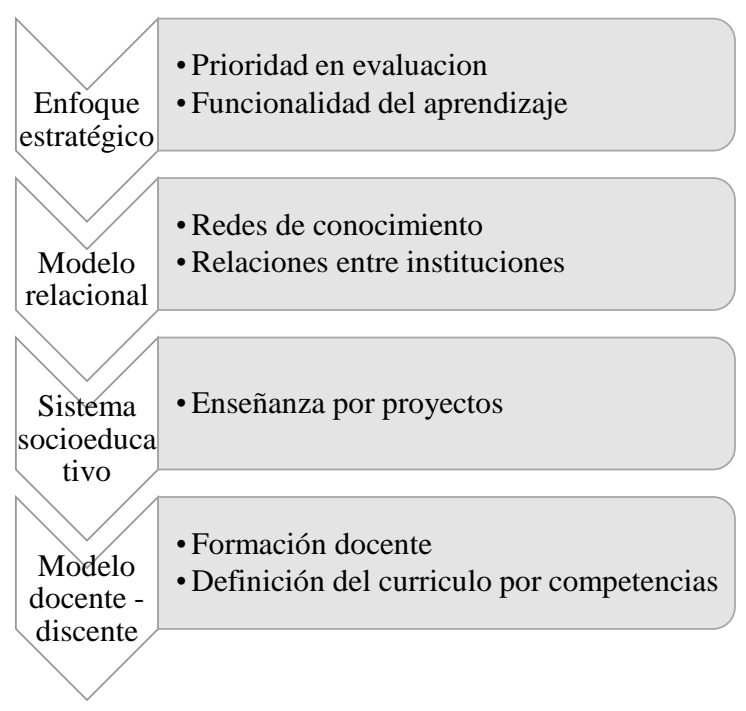

Figura 5: Modelos metodológicos básicos.

Papel de las familias. Como componente básico de la comunidad educativa, las familias también desarrollarán una actividad importante en el proceso educativo de los alumnos. Para ello, se indican una serie de principios a seguir para desarrollar una actitud de trabajo con los profesionales del centro educativo. Estas serían:

- Participar y colaborar en el establecimiento y seguimiento de la metodología de trabajo en el centro y en casa. Controlando los distintos aspectos a tener en cuenta para desarrollar el aprendizaje de forma eficiente.

- Fomentar y facilitar la mejora de aspectos como el autoconcepto y la autoestima, así como fortalecer la atención y la motivación para alcanzar las distintas metas planteadas. Con ello se pretende conseguir una estabilidad emocional y afectiva que redunde en el interés por los estudios.

- Además, será imprescindible establecer vías de comunicación y participación efectivas entre el centro y las familias para que exista sintonía entre las actividades realizadas en el aula y fuera de ella. A ello se le puede sumar la participación de las familias en actividades propias del centro en busca de una colaboración activa donde se compartan acciones ante los problemas que pudieran aparecer.

\section{Discusión}

Si tuviéramos que destacar una idea en materia de atención a la diversidad que se repita a lo largo del texto que se ha descrito bien podría ser la señalada para la comunidad autónoma de Galicia en Fidalgo y Robledo (2010 p. 177) "Actuaciones educativas dirigidas a dar respuesta a las diferentes capacidades, ritmos y estilos de aprendizaje.” Encontrando que el principal propósito de las indicaciones que se han analizado es dar respuesta a la atención a la diversidad desde todas sus vertientes. Además, un factor fundamental a considerar en las DEA es que existe una posibilidad de mejora, y que si se incide oportunamente con los recursos y las estrategias necesarios se producirá un desarrollo efectivo en el alumno. Esto teniendo su origen en una necesidad que debe ser cubierta, se transforma en exigencia y deber de adaptar las enseñanzas al alunado con necesidades específicas de apoyo educativo. (Millá, 2006; Fidalgo y Robledo, 2010; Castaño Calle, 2013). En ello, los docentes, como figura reguladora del especio educativo, tienen la responsabilidad de ejercer una atención a la diversidad activa y de constante mejora, atendiendo a los distintos procesos dentro y fuera de aula (Cardinale y cuevas, 2013; Contreras, Torres, Araque y Herrera, 2016).

Esto, como todo proceso de intervención, debe llevarse a cabo, con la sistematicidad suficiente que desarrolle una práctica docente rigurosa en la actuación ante la diversidad del alumnado. Para ello, las distintas pruebas normalizadas de valoración son una herramienta de primera mano para los equipos de orientación y profesorado de apoyo (Piñas, Niño y Silvestre, 2012; Rodríguez, Martínez y Castro, 2014). Con ello, no se pretende ejercer una labor de intervención fría y distanciada de la realidad, sino conseguir adaptar con mayor precisión las distintas medidas a tomar con posterioridad, y que las estrategias metodológicas sean lo más acertadas para la evolución del alumno.

Otro aspecto en el que se incide de forma concisa es el referente a la relación familia - escuela. La investigación emergente en este ámbito da por justificada la importancia de los procesos de relación entre la familia y la escuela (Ramón y Sánchez, 2013; Parada-Gañete, 2015). Esto, es un hecho que se demuestra al encontrar la demanda de colaboración y participación en la vida de centro por parte de las familias de la comunidad educativa en su globalidad, no solo las de los alumnos con NEAE. Con ello se pretende que la mayor implicación de las familias en el desarrollo de la vida de aula y de centro se utilice como beneficio para el desarrollo de un trabajo holístico hacia el alumnado.

Por último, señalar algo que no se ha encontrado en este proceso de revisión y que así lo indica Goikoetxea (2012), "tenemos lagunas de conocimiento y carencia de programas y metodologías que posibiliten una enseñanza basada en la investigación científica de los niños con DEA". Esta autora habla de unas lagunas de conocimiento que se ven igualmente destacadas en Guzmán, Correa, Arvelo y Abreu (2015), donde también se indica como aspecto relevante la existencia de esta falta de conocimiento y la falta de dirección en este sentido. Para la mejora de este aspecto en el proceso, es necesario que el docente lleve a cabo procesos de investigación-acción, lo que posibilita otro de los aspectos señalados de forma recurrente en este texto, la búsqueda de la mejora continua de las distintas prácticas de atención a la diversidad.

\section{Referencias}

A.P.A. (2000). Diagnostics and statistics manual of mental disorders: DSM-IV-TR. Washington: APA.

Bisquerra, R. (2016). Metodología de la investigación educativa. Madrid: La Muralla 
Cardinale, L., \& Cuevas, V. (2013). ¿Qué sujeto de aprendizaje?: Recuperar el pasado para pensar la intervención psicopedagógica. Pilquen - Sección Psicopedagogía, 10, 2-8.

Castaño Calle, R. (2010). La atención educativa integral a los alumnos con necesidades específicas de apoyo educativo: actitudes, valores y normas. Hekademos: revista educativa digital, 7, 23-42.

Contreras, B. E. M., Torres, K. F., Araque, B. L., \& Herrera, D. M. G. (2016). Dificultades específicas del aprendizaje en estudiantes de primaria de una escuela pública en Cartagena. Areté, 16(1), 79-89.

Fidalgo Redondo, R., \& Robledo Ramón, P. (2010). El ámbito de las dificultades específicas de aprendizaje en España a partir de la Ley Orgánica de Educación. Papeles del psicólogo, 31(2), 171-182.

Goikoetxea, E. (2012). Las dificultades específicas de aprendizaje en el albor del siglo XXI. Relieve: Revista ELectrónica de Investigación y EValuación Educativa, 18(1), 2.

Guzmán Rosquete, R., Correa Piñero, A. D., Arvelo Rosales, C. N., \& Abreu Pérez, B. (2015). Conocimiento del profesorado sobre las dificultades específicas de aprendizaje en lectura y escritura. Revista de Investigación Educativa, 33(2), 289-302. https://doi.org/10.6018/rie.33.2.211101

Millá, M. G. (2006). Atención temprana de las dificultades de aprendizaje. Revista de neurología, 42(2), 153-156.

OMS (1992). CIE-10. Décima revisión d la clasificación internacional de las enfermedades. Madrid: Medicor.

Parada-Gañete, A. (2015). Relación familia-escola: caso concreto do alumnado con Dificultades de Aprendizaje. REIPE, Revista de estudios e investigación en psicología y educación. 11,

Piñas, M. L., Niño, J. A. R., \& Silvestre, L. Z. (2012). Proceso cognitivo y metacognitivo en prescolares con riesgo de dificultades de aprendizaje de las matemáticas según sus padres. Fòrum de Recerca, 17, 829-838.

Ramón, P. R., \& Sánchez, J. N. G. (2013). La implicación y satisfacción educativa de las familias de alumnado con y sin dificultades específicas de aprendizaje. Revista de psicodidáctica, 18(1), 137-156.

Rodríguez, L. F. G., Martínez, A. M., \& Castro, E. Z. (2014). Diagnóstico de niños con TEL y dificultades de aprendizaje con la ITPA-3. Areté, 14, 183-194.

Strnadová, I. (2006). Estrés y adaptación en familias con niños con dificultades específicas de aprendizaje. Revista complutense de educación, 17(2), 35-50.

Xunta de Galicia. (2008). Alumnado con trastornos de aprendizaxe. Colección: Orientación e respostas educativas. Tórculo edicións. 